\title{
Chemical and Radiochemical Background Concentrations of Geologic Materials Beneath Fermilab
}

\author{
P.M. KESICH, K. VAZIRI, N. GROSSMAN \\ FERMI NATIONAL ACCELERATOR LABORATORY
}

FEKRUARY 22, 2000

\begin{abstract}
This document consists of a group of tables containing chemical and radiochemical analytical results of samples of geologic materials at Fermi National Accelerator Laboratory. The results are for materials from ground surface to approximately 375 feet below ground surface. The results are from materials obtained from previously undisturbed areas and represent background values. Material for analyses were taken from boreholes drilled as part of the NuMI ground water monitoring network.
\end{abstract}


The current methodology in use at Fermilab for estimating radionuclide production in soil or rock and migration in the saturated zone to the regulated ground water, is the Concentration Model. Malensek, et al. (Fermilab-TM-1851), proposed this model in 1993 as a new approach over the previously used Single Resident Well Model (Jonckheere, December 1978). EP Note 8 (Cossairt, December 1994), summarized the principal conclusions and made a specific recommendation to the Fermilab Director concerning the application of the results to the future design of targets and absorbers at Fermilab. Subsequently, clarification and modification of aspects of the model were done (Cossairt et al., EP Note 17) and its application for unique conditions addressed (Grossman et al., Fermilab-TM-2.103).

One of the parameters of the Concentration Model is the avcrage star density $(<\mathrm{S}\rangle)$ in the geologic material outside of the targeting enclosure. This value is determined from a Monte Carlo computer program for the simulation of hadronic and electromagnetic cascades. Input to the program can be for generic material or can include specific chemical compositions. Part of the output of this program is tables and contour plots of star density for the composition and geometry of the area. In the determination of shielding and operational design, the composition of the formation outside of the production area may be an important factor when calculating residual dose rates.

The chemical composition of soil outside of a beam enclosure has been made for limited cases along with activation products from soil and rock (Fairman, et el. 1970, Awschalom 1971, Borak et el. 1972, Gollon 1978, Cupps 1998). Chemical analyses of Illinois limestones and dolomites have been compiled to present information regarding the various rock formations for use in evaluating the chemical character of these resources by the Illinois State Geological Survey (1957). This report focuses on the background chemical and radiochemical composition of samples of geologic materials from the ground surface to approximately 375 feet below ground surface $^{1}$. The materials consist of the consolidated bedrock formations that make up the upper bedrock aquifer and the unconsolidated glacial deposits that confine them. Samples of geologic material were taken from cores that were drilled during the initial ground water monitoring network installation for the NuMI project. Radiochemical analysis was performed at the onsite Activation Analysis Laboratory ${ }^{2}$ and chemical analysis was performed at an offsite vendor, Actlabs-Skyline, Tucson, $\mathrm{AZ}^{3}$. Detailed descriptions of the stratigraphy are documented (Rust 1996, STS 1997 and Earth Tech 1999.) With this information on the background chemical and radiochemical composition of geologic materials, comparisons can he made between dose rates obtained using "standard" geologic material compositions and the actual chemical compositions.

\footnotetext{
${ }^{1}$ The last sample was taken from 340.5 feet below ground surface, however the borehole extended to 375 feet with a similar lithology from 340 to the bottom of the core.

${ }^{2}$ Radiochemical analysis was for gammas only. The samples were crushed and transferred to a poly bottle prior to counting. Each sample was then counted for 14,400 seconds at $1.2234 \mathrm{~cm}$ on detector $\mathrm{Ge}(\mathrm{Li}) \# 1$. The total gross counts for each sample was measured in the energy range of 20-3050 keV. The hackground counts were subtracted from the sample counts prior to the activities being calculated. Activities were corrected back to the time of sampling. Water was not driven off of the samples for tritium analysis.

${ }^{3}$ Samples were analyzed at research grade using Inductively Coupled Plasma Optical Emmision Spectroscopy (ICPOES), Instrumental Neutron Activation Analysis (INAA), and Inductively Coupled Plasma Mass Spectroscopy (ICP-MS) technologies.
} 


\section{References:}

1. Awschalom, M., "Calculation of the Radionuclide Production in the Surroundings of the NAL Neutrino Laboratory." Intcrnal Rcport TM-292-A, Fermi National Accelerator Laboratory, Batavia, IL March 1971.

2. Borak, T. B., M. Awschalom, W. Fairman, F. Iwami and J. Sedlet, "The Underground Migration of Radionuclides Produced in Soil near High Energy Proton Accelerators." Health Physics, Vol. 23 (Nov), pp. 679-687, 1972.

3. Cossairt, J. D., "Usc of a Concentration-Based Model for Calculating the Radioactivation of Soil and Groundwater at Fermilab." Internal Report Environmental Protection Note 8, Fermi National Accelerator Laboratory, Batavia, IL, December 1994.

4. Cossairt, J. D., A. J. Elwyn, P. Kesich, A. Malensek, N. Mokhov, and A. Wehmann, "The Concentration Model Revisited." Internal Report Environmental Protection Note 17, Fermi National Accelerator Laboratory, Batavia, IL, June 1999.

5. Cupps, V., "Estimation of Leaching Parameters from NuMI Rock Boring Sample Data." Internal Report Radiation Physics Note 136, January 2000.

6. Earth Tech, "Summarization of NuMI Piezometer Installation Project." December 1999.

7. Fairman, W., W. Hranka, J. Sedlet, M. Awschalom, and T. B. Borak, "The Extraction and Transport of Radionuclides Produced in Soil by High Energy Hadrons." Internal Report TM247, Fermi National Accelerator Laboratory, Batavia, IL, June 1970.

8. Golon, P. J., "Production of Radioactivity by Particle Accelerators." Internal Report TM609, Fermi National Accelerator Laboratory, Batavia, IL, May 1976.

9. Grossman, N., F. Breen, S. Childress, P. Kesich, C. Laughton, A. Wehmann, "Refïnement of Groundwater Protection for the NuMI Project." Internal Report TM-2103, Fermi National Accelerator Laboratory February 2000.

10. Illinois State Geological Survey, "Chemical Analysis of Illinois Limestones and Dolomites." Report of Investigations 200, 1957.

11. Jonckheere, A. M., "Aquifer Dilution Factors of Ground Water Activity Produced Around Fermilab Targets and Dumps." Internal Report TM-838, Fermi National Accelerator Laboratory, Batavia, IL, December 1978.

12. Malensek, A. J., A. A. Wehmann, A. J. Elwyn, K. J. Moss, and P. M. Kesich, "Groundwater Migration of Radionuclides at Fermilab." Internal Report TM-1851, Fermi National Accelerator Laboratory, Batavia, IL, August 1993. 
13. RUST Environment and Infrastructure, "NS1 and NS2 Hydrogeologic Site Characterization and Monitoring Well Installation." December 1996.

14. STS Consultants, Ltd., "Hydrogeological Evaluation Report Neutrino Main Injector (NuMI)." April 1997. 


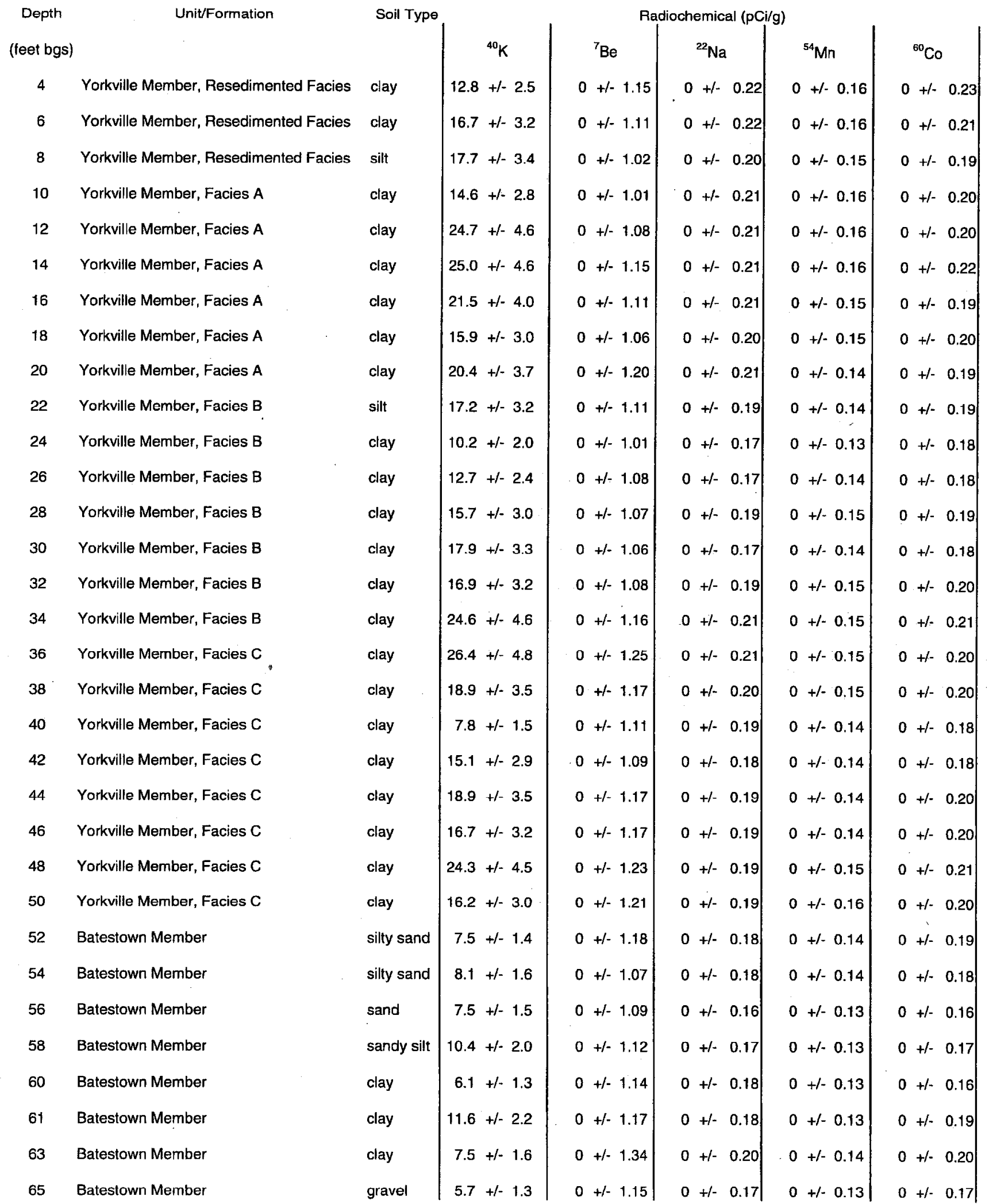




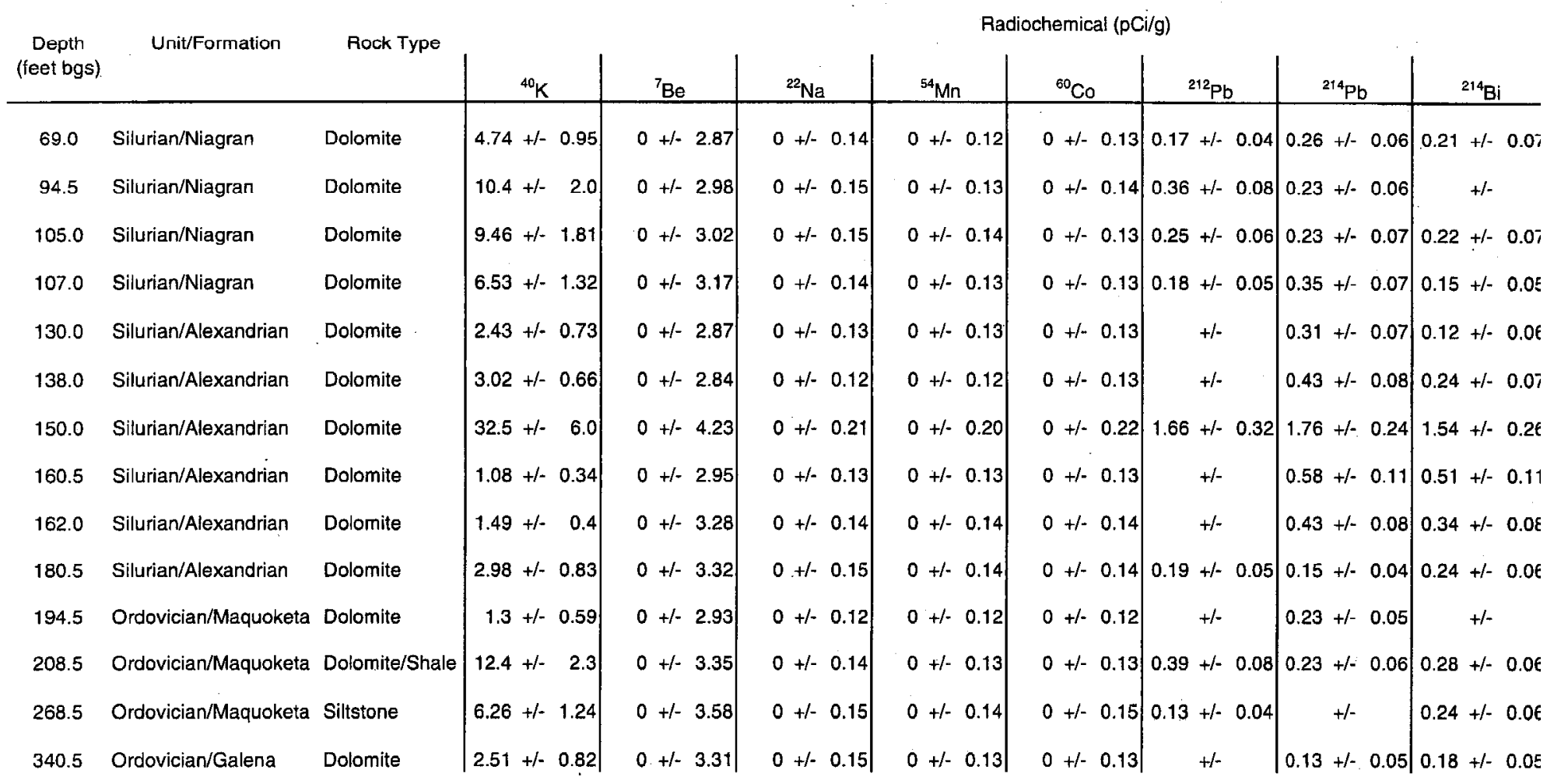

bgs $=$ below ground surface (ground surface is approximately 740 feet Mean Sea Level)

uncertainties are calculated from error values of efficiency, absortion correction and geometry 
Table of Background Trace Elements from Soil and Rock Samples using Fusion Inductively Coupled Plasma Mass ؛

\begin{tabular}{|c|c|c|c|c|c|c|c|c|c|c|c|}
\hline $\begin{array}{c}\text { Depth } \\
\text { (feet bgs) }\end{array}$ & Unit/Formation & Material Type & $\begin{array}{r}\mathrm{Ga} \\
\mathrm{ppm}\end{array}$ & $\begin{array}{r}\mathrm{Ge} \\
\mathrm{ppm}\end{array}$ & $\begin{array}{r}\mathrm{Rb} \\
\mathrm{ppm}\end{array}$ & $\begin{array}{r}\mathrm{Sr} \\
\mathrm{ppm}\end{array}$ & $\begin{array}{r}\mathrm{Y} \\
\mathrm{ppm}\end{array}$ & $\begin{array}{r}\mathrm{Zr} \\
\mathrm{ppm}\end{array}$ & $\begin{array}{r}\mathrm{Nb} \\
\mathrm{ppm}\end{array}$ & $\begin{array}{r}\text { Mo } \\
\text { Ppm }\end{array}$ & $\begin{array}{r}\text { In } \\
\text { ppm }\end{array}$ \\
\hline $7-11$ & Quaternary & Diamicton & -1 & -0.5 & 13 & 70 & 6.7 & 40 & 2.1 & -2 & -0.1 \\
\hline $11-14$ & Quaternary & Diamicton & -1 & -0.5 & 29 & 82 & 8.9 & 40 & 4 & -2 & -0.1 \\
\hline $28-35$ & Quaternary & Resedimented & 2 & -0.5 & 32 & 86 & 8.9 & 39 & 4.7 & -2 & -0.1 \\
\hline $39-42$ & Quaternary & Diamicton & 1 & -0.5 & 25 & 104 & 10.4 & 34 & 3.9 & -2 & -0.1 \\
\hline $42-53$ & Quaternary & Diamicton & -1 & -0.5 & 5 & 89 & 4.1 & 19 & 1 & -2 & -0.1 \\
\hline $54-58$ & Quaternary & Diamicton & -1 & -0.5 & 15 & 99 & 6.5 & 26 & 2.6 & -2 & -0.1 \\
\hline $58-65$ & Quaternary & Diamicton & 18 & 1.7 & 162 & 112 & 22.5 & 236 & 21.4 & -2 & -0.1 \\
\hline 69.0 & Silurian/Niagran & Dolomite & -1 & -0.5 & -2 & 76 & 6.3 & 16 & 0.7 & -2 & -0.1 \\
\hline 94.5 & Silurian/Niagran & Dolomite & -1 & -0.5 & 4 & 68 & 6.1 & 15 & 1 & -2 & -0.1 \\
\hline 105.0 & Silurian/Niagran & Dolomite & -1 & -0.5 & 11 & 64 & 7 & 20 & 1.7 & -2 & -0.1 \\
\hline 107.0 & Silurian/Niagran & Dolomite & -1 & -0.5 & 3 & 50 & 8.6 & 15 & 0.6 & -2 & -0.1 \\
\hline 130.0 & Silurian/Alexandrian & Dolomite & -1 & -0.5 & 47 & 88 & 12.8 & 54 & 5.7 & -2 & -0.1 \\
\hline 138.0 & Silurian/Alexandrian & Dolomite & -1 & -0.5 & 53 & 89 & 12.8 & 62 & 5.8 & -2 & -0.1 \\
\hline 150.0 & Silurian/Alexandrian & Dolomite & -1 & -0.5 & 4 & 55 & 7.2 & 12 & 0.8 & -2 & -0.1 \\
\hline 160.5 & Silurian/Alexandrian & Dolomite & 4 & 1 & 88 & 82 & 20.3 & 115 & 9.5 & 5 & -0.1 \\
\hline 162.0 & Silurian/Alexandrian & Dolomite & 6 & 0.9 & 86 & 89 & 19 & 133 & 9.4 & 4 & -0.1 \\
\hline 180.5 & Silurian/Alexandrian & Dolomite & 9 & 1 & 106 & 95 & 20.5 & 114 & 11.5 & 5 & -0.1 \\
\hline 194.5 & Ordovician/Maquoketa & Dolomite & 5 & 0.6 & 68 & 95 & 15.1 & 107 & 6.8 & 3 & -0.1 \\
\hline 208.5 & Ordovician/Maquoketa & Dolomite/Shale & 10 & 1 & 125 & 121 & 21 & 130 & 11.3 & 5 & -0.1 \\
\hline 268.5 & Ordovician/Maquoketa & Siltstone & -1 & 0.7 & 36 & 103 & 12.9 & 96 & 4.2 & -2 & -0.1 \\
\hline 340.5 & Ordovician/Galena & Dolomite & -1 & -0.5 & 35 & 106 & 12.4 & 115 & 5.2 & -2 & -0.1 \\
\hline
\end{tabular}

Negative values equal not detected at that lower limit.

bgs = below ground surface (ground surface is approximately 740 feet Mean Sea Level) 


\begin{tabular}{|c|c|c|c|c|c|c|c|c|c|c|c|c|c|c|}
\hline $\begin{array}{c}\text { Depth } \\
\text { (feet bgs) }\end{array}$ & Unit/Formation & Material Type & $\begin{array}{r}\mathrm{La} \\
\mathrm{ppm} \\
\end{array}$ & $\begin{array}{r}\mathrm{Ce} \\
\mathrm{ppm} \\
\end{array}$ & $\begin{array}{r}\text { Pr } \\
\text { ppm } \\
\end{array}$ & $\begin{array}{r}\mathrm{Nd} \\
\mathrm{ppm} \\
\end{array}$ & $\begin{array}{r}\mathrm{Sm} \\
\mathrm{ppm} \\
\end{array}$ & $\begin{array}{r}\text { Eu } \\
\text { ppm }\end{array}$ & $\begin{array}{r}\mathrm{Gd} \\
\mathrm{ppm} \\
\end{array}$ & $\begin{array}{r}\mathrm{Tb} \\
\mathrm{ppm} \\
\end{array}$ & $\begin{array}{r}\text { Dy } \\
\text { ppm } \\
\end{array}$ & $\begin{array}{r}\text { Ho } \\
\text { ppm } \\
\end{array}$ & $\begin{array}{r}\text { Er } \\
\mathrm{ppm} \\
\end{array}$ & $\begin{array}{r}\mathrm{Tm} \\
\text { ppm }\end{array}$ \\
\hline $7-11$ & Quaternary & Diamicton & 8.79 & 10 & 1.61 & 6.15 & 1.07 & 0.248 & 1.09 & 0.16 & 0.94 & 0.2 & 0.56 & 0.077 \\
\hline $11-14$ & Quaternary & Diamicton & 15 & 22.9 & 2.63 & 9.65 & 1.68 & 0.357 & 1.5 & 0.24 & 1.36 & 0.28 & 0.81 & 0.111 \\
\hline $28-35$ & Quaternary & Resedimented & 13.6 & 17.8 & 2.36 & 8.63 & 1.62 & 0.334 & 1.42 & 0.21 & 1.23 & 0.26 & 0.76 & 0.108 \\
\hline $39-42$ & Quaternary & Diamicton & 18.4 & 21 & 2.76 & 10.1 & 1.77 & 0.372 & 1.73 & 0.26 & 1.43 & 0.29 & 0.81 & 0.11 \\
\hline $42-53$ & Quaternary & Diamicton & 6.62 & 8 & 1.08 & 4.21 & 0.72 & 0.172 & 0.7 & 0.11 & 0.59 & 0.13 & 0.34 & 0.045 \\
\hline $54-58$ & Quaternary & Diamicton & 9.18 & 12.6 & 1.57 & 6.14 & 1.03 & 0.223 & 0.97 & 0.17 & 0.9 & 0.19 & 0.54 & 0.075 \\
\hline $58-65$ & Quaternary & Diamicton & 49.2 & 78.3 & 8.7 & 30.3 & 4.44 & 0.869 & 3.46 & 0.54 & 3.37 & 0.74 & 2.46 & 0.379 \\
\hline 69.0 & Silurian/Niagran & Dolomite & 3.18 & 5.6 & 0.66 & 2.9 & 0.7 & 0.178 & 0.78 & 0.14 & 0.79 & 0.15 & 0.43 & 0.056 \\
\hline 94.5 & Silurian/Niagran & Dolomite & 3.7 & 6.6 & 0.79 & 3.11 & 0.76 & 0.178 & 0.88 & 0.14 & 0.82 & 0.16 & 0.45 & 0.062 \\
\hline 105.0 & Silurian/Niagran & Dolomite & 6.1 & 11.2 & 1.33 & 5.43 & 1.17 & 0.249 & 1.13 & 0.19 & 1 & 0.2 & 0.55 & 0.074 \\
\hline 107.0 & Silurian/Niagran & Dolomite & 5.41 & 9 & 1.29 & 5.28 & 1.11 & 0.252 & 1.29 & 0.18 & 1.05 & 0.23 & 0.64 & 0.09 \\
\hline 130.0 & Silurian/Alexandrian & Dolomite & 15 & 25.6 & 3.11 & 12.2 & 2.4 & 0.518 & 2.11 & 0.36 & 1.98 & 0.42 & 1.27 & 0.178 \\
\hline 138.0 & Silurian/Alexandrian & Dolomite & 15.2 & 25.5 & 3.11 & 12.3 & 2.29 & 0.505 & 2.16 & 0.34 & 2 & 0.41 & 1.22 & 0.184 \\
\hline 150.0 & Silurian/Alexandrian & Dolomite & 5.73 & 6.3 & 0.84 & 3.51 & 0.67 & 0.151 & 0.74 & 0.13 & 0.8 & 0.17 & 0.51 & 0.074 \\
\hline 160.5 & Silurian/Alexandrian & Dolomite & 26.6 & 52.5 & 5.85 & 21.9 & 4.1 & 0.913 & 3.92 & 0.63 & 3.63 & 0.73 & 2.06 & 0.303 \\
\hline 162.0 & Silurian/Alexandrian & Dolomite & 22.9 & 45.1 & 5.01 & 19.1 & 3.72 & 0.801 & 3.17 & 0.54 & 3.1 & 0.66 & 1.97 & 0.274 \\
\hline 180.5 & Silurian/Alexandrian & Dolomite & 27.2 & 54.4 & 6.05 & 22.6 & 4.25 & 0.898 & 3.84 & 0.62 & 3.65 & 0.73 & 2.1 & 0.306 \\
\hline 194.5 & Ordovician/Maquoketa & Dolomite & 18.1 & 35.3 & 4.03 & 15.6 & 2.98 & 0.691 & 2.71 & 0.45 & 2.5 & 0.53 & 1.55 & 0.223 \\
\hline 208.5 & Ordovician/Maquoketa & Dolomite/Shale & 29.1 & 57.2 & 6.24 & 23.3 & 4.22 & 0.948 & 3.73 & 0.62 & 3.49 & 0.74 & 2.16 & 0.32 \\
\hline 268.5 & Ordovician/Maquoketa & Siltstune & 12 & 23 & 2.86 & 11.5 & 2.39 & 0.603 & 2.29 & 0.41 & 2.13 & 0.45 & 1.31 & 0.184 \\
\hline 340.5 & Ordovician/Galena & Dolomite & 14.2 & 26.3 & 3.17 & 12.1 & 2.26 & 0.564 & 2.19 & 0.36 & 2.08 & 0.42 & 1.27 & 0.18 \\
\hline
\end{tabular}

Negative values equal not detected at that lower limit.

bgs = below ground surface (ground surface is approximately 740 feet Mean Sea Level) 
Table of Background Trace Elements from Soil and Rock Samples using Fusion Inductively Coupled Plasma Mass Spectroscopy

\begin{tabular}{|c|c|c|c|c|c|c|c|c|c|c|c|}
\hline $\begin{array}{c}\text { Depth } \\
\text { (feet bgs) }\end{array}$ & Unit/Formation & Material Type & $\begin{array}{r}\mathrm{Yb} \\
\mathrm{ppm} \\
\end{array}$ & $\begin{array}{r}\mathrm{Lu} \\
\mathrm{ppm}\end{array}$ & $\begin{array}{r}\mathrm{Hf} \\
\mathrm{ppm} \\
\end{array}$ & $\begin{array}{r}\mathrm{Ta} \\
\mathrm{ppm} \\
\end{array}$ & $\begin{array}{r}W \\
\mathrm{ppm} \\
\end{array}$ & $\begin{array}{r}\mathrm{Tl} \\
\mathrm{ppm} \\
\end{array}$ & $\begin{array}{r}\mathrm{Bi} \\
\mathrm{ppm} \\
\end{array}$ & $\begin{array}{r}\text { Th } \\
\text { ppm }\end{array}$ & $\begin{array}{r}\mathrm{U} \\
\text { ppm }\end{array}$ \\
\hline $7-11$ & Quaternary & Diamicton & 0.46 & 0.071 & 0.7 & -0.1 & 0.3 & -0.05 & -0.06 & 1.19 & 0.24 \\
\hline $11-14$ & Quaternary & Diamicton & 0.7 & 0.116 & 1 & 0.2 & 1.1 & 0.11 & -0.06 & 3.21 & 0.34 \\
\hline $28-35$ & Quaternary & Resedimented & 0.65 & 0.103 & 1 & 0.3 & 0.9 & 0.21 & -0.06 & 3.77 & 0.41 \\
\hline $39-42$ & Quaternary & Diamicton & 0.69 & 0.099 & 0.8 & 0.2 & 0.9 & 0.11 & -0.06 & 3.64 & 0.34 \\
\hline $42-53$ & Quaternary & Diamicton & 0.24 & 0.034 & 0.4 & -0.1 & -0.2 & -0.05 & -0.06 & 0.7 & 0.43 \\
\hline $54-58$ & Quaternary & Diamicton & 0.45 & 0.072 & 0.6 & 0.1 & 0.3 & -0.05 & -0.06 & 1.34 & 0.65 \\
\hline $58-65$ & Quaternary & Diamicton & 2.6 & 0.413 & 6.5 & 1.4 & 3.2 & 0.55 & 0.19 & 10.7 & 5.83 \\
\hline 69.0 & Silurian/Niagran & Dolomite & 0.35 & 0.046 & 0.2 & -0.1 & -0.2 & -0.05 & -0.06 & 0.56 & 1.1 \\
\hline 94.5 & Silurian/Niagran & Dolomite & 0.36 & 0.055 & 0.3 & -0.1 & 0.2 & -0.05 & -0.06 & 0.73 & 0.99 \\
\hline 105.0 & Silurian/Niagran & Dolomite & 0.46 & 0.073 & 0.5 & -0.1 & 0.2 & -0.05 & -0.06 & 1.19 & 0.51 \\
\hline 107.0 & Silurian/Niagran & Dolomite & 0.5 & 0.071 & 0.3 & -0.1 & -0.2 & -0.05 & -0.06 & 0.48 & 0.42 \\
\hline 130.0 & Silurian/Alexandrian & Dolomite & 1.11 & 0.17 & 1.5 & 0.4 & 0.7 & 0.11 & -0.06 & 3.4 & 0.74 \\
\hline 138.0 & Silurian/Alexandrian & Dolomite & 1.17 & 0.169 & 1.6 & 0.4 & 0.8 & 0.16 & -0.06 & 3.4 & 0.75 \\
\hline 150.0 & Silurian/Alexandrian & Dolomite & 0.4 & 0.054 & 0.3 & -0.1 & 0.2 & -0.05 & -0.06 & 0.48 & 0.51 \\
\hline 160.5 & Silurian/Alexandrian & Dolomite & 2.01 & 0.302 & 3.5 & 0.7 & 1.6 & 0.25 & -0.06 & 6.82 & 3.24 \\
\hline 162.0 & Silurian/Alexandrian & Dolomite & 1.76 & 0.27 & 3.5 & 0.6 & 1.7 & 0.66 & -0.06 & 5.89 & 3.29 \\
\hline 180.5 & Silurian/Alexandrian & Dolomite & 2.05 & 0.307 & 3.2 & 0.8 & 2 & 0.35 & -0.06 & 7.65 & 3.85 \\
\hline 194.5 & Ordovician/Maquoketa & Dolomite & 1.38 & 0.222 & 2.8 & 0.5 & 1.3 & 0.37 & -0.06 & 4.7 & 2.71 \\
\hline 208.5 & Ordovician/Maquoketa & Dolomite/Shale & 2.07 & 0.309 & 3.7 & 0.8 & 1.9 & 0.29 & -0.06 & 7.96 & 4.64 \\
\hline 268.5 & Ordovician/Maquoketa & Siltstone & 1.17 & 0.172 & 2.5 & 0.3 & 1.3 & 0.26 & -0.06 & 2.6 & 1.04 \\
\hline 340.5 & Urdovician/Galena & Dolomite & 1.17 & 0.179 & 3 & 0.3 & 0.7 & 0.2 & -0.06 & 3.17 & 1.16 \\
\hline
\end{tabular}

Negative values equal not detected at that lower limit.

bgs = below ground surface (ground surface is approximately 740 feet Mean Sea Level) 
Table of Background Chemicals from Soil and Rock Samples using Instrumental Neutron Activation Analysis

\begin{tabular}{|c|c|c|c|c|c|c|c|c|c|c|c|c|c|c|}
\hline $\begin{array}{c}\text { Depth } \\
\text { (feet bgs) }\end{array}$ & Unit/Formation & Material Type & $\begin{array}{r}\mathrm{Au} \\
\mathrm{ppb}\end{array}$ & $\begin{array}{r}\text { As } \\
\mathrm{ppm}\end{array}$ & $\begin{array}{r}\mathrm{Br} \\
\mathrm{ppm} \\
\end{array}$ & $\begin{array}{r}\mathrm{Co} \\
\mathrm{ppm} \\
\end{array}$ & $\begin{array}{r}\mathrm{Cr} \\
\mathrm{ppm} \\
\end{array}$ & $\begin{array}{r}\mathrm{Hg} \\
\mathrm{ppm}\end{array}$ & $\begin{array}{r}\mathrm{Ir} \\
\mathrm{ppb} \\
\end{array}$ & $\begin{array}{r}\text { Mo } \\
\text { ppm } \\
\end{array}$ & $\begin{array}{r}\mathrm{Sb} \\
\mathrm{ppm} \\
\end{array}$ & $\begin{array}{r}\mathrm{Sc} \\
\mathrm{ppm} \\
\end{array}$ & $\begin{array}{r}\text { Se } \\
\mathrm{ppm}\end{array}$ & $\begin{array}{r}\text { Mass } \\
\mathrm{g} \\
\end{array}$ \\
\hline $7-11$ & Quaternary & Diamicton & $<2$ & 10 & 2.9 & 13.1 & 58.7 & $<1$ & $<2$ & 7 & 0.8 & 10.4 & $<0.5$ & 1.637 \\
\hline $11-14$ & Quaternary & Diamicton & $<2$ & 9 & 3.6 & 12.8 & 52.1 & $<1$ & $<2$ & 7 & 0.7 & 9.44 & $<0.5$ & 1.433 \\
\hline $28-35$ & Quaternary & Resedimented & $<2$ & 9 & 2.2 & 16.7 & 74.1 & $<1$ & $<2$ & 7 & 0.9 & 12.8 & $<0.5$ & 1.285 \\
\hline $39-42$ & Quaternary & Diamicton & $<2$ & 9 & 4.2 & 9.6 & 43.6 & $<1$ & $<2$ & 4 & 0.5 & 7.68 & $<0.5$ & 1.44 \\
\hline $42-53$ & Quaternary & Diamicton & $<2$ & 9 & 2.1 & 16.1 & 76.7 & $<1$ & $<2$ & 10 & 0.7 & 12.9 & $<0.5$ & 1.317 \\
\hline $54-58$ & Quaternary & Diamicton & $<2$ & 5 & 6.1 & 9.6 & 41.4 & $<1$ & $<2$ & $<2$ & 0.3 & 7.35 & $<0.5$ & 1.853 \\
\hline $58-65$ & Quaternary & Diamicton & $<2$ & 5 & 7.1 & 5.4 & 26.3 & $<1$ & $<2$ & $<2$ & 0.3 & 4.29 & $<0.5$ & 1.736 \\
\hline 69.0 & Silurian/Niagran & Dolomite & 2 & $<1$ & 20.4 & 1.6 & 45.8 & $<1$ & $<2$ & $<2$ & $<0.1$ & 1.72 & $<0.5$ & 1.497 \\
\hline 94.5 & Silurian/Niagran & Dolomite & $<2$ & 2 & 16.1 & 6.6 & 11.4 & $<1$ & $<2$ & $<2$ & 0.4 & 3.5 & $<0.5$ & 1.623 \\
\hline 105.0 & Silurian/Niagran & Dolomite & 2 & 1 & 20.5 & 2.4 & 12.3 & $<1$ & $<2$ & $<2$ & 1 & 4.38 & $<0.5$ & 1.691 \\
\hline 107.0 & Silurian/Niagran & Dolomite & 3 & 1 & 26.4 & 2.1 & 9.4 & $<1$ & $<2$ & $<2$ & 0.3 & 4.43 & $<0.5$ & 1.594 \\
\hline 130.0 & Silurian/Alexandrian & Dolomite & $<2$ & $<1$ & 30.2 & 1.7 & 3.2 & $<1$ & $<2$ & $<2$ & $<0.1$ & 0.81 & $<0.5$ & 1.509 \\
\hline 138.0 & Silurian/Alexandrian & Dolomite & $<2$ & $<1$ & 19.1 & 2 & 9.3 & $<1$ & $<2$ & $<2$ & $<0.1$ & 1.53 & $<0.5$ & 1.778 \\
\hline 150.0 & Silurian/Alexandrian & Dolomite & $<2$ & 4 & 1.5 & 4.4 & 85.8 & $<1$ & $<2$ & $<2$ & 0.7 & 13.1 & $<0.5$ & 1.893 \\
\hline 160.5 & Silurian/Alexandrian & Dolomite & $<2$ & 2 & 7.1 & 1.9 & 4.5 & $<1$ & $<2$ & 2 & 0.2 & 1 & $<0.5$ & 1.712 \\
\hline 162.0 & Silurian/Alexandrian & Dolomite & 2 & 1 & 6.8 & 1.9 & 4.8 & $<1$ & $<2$ & $<2$ & 0.2 & 0.79 & $<0.5$ & 1.762 \\
\hline 180.5 & Silurian/Alexandrian & Dolomite & $<2$ & $<1$ & 3 & 0.9 & 9.8 & $<1$ & $<2$ & $<2$ & $<0.1$ & 1.33 & $<0.5$ & 1.88 \\
\hline 194.5 & Ordovician/Maquoketa & Dolomite & $<2$ & 1 & 4.2 & 3.7 & 2.8 & $<1$ & $<2$ & $<2$ & $<0.1$ & 0.76 & $<0.5$ & 1.775 \\
\hline 208.5 & Ordovician/Maquoketa & Dolomite/Shale & $<2$ & $<1$ & 2.8 & 2.9 & 22 & $<1$ & $<2$ & $<2$ & 0.2 & 4.95 & $<0.5$ & 1.694 \\
\hline 268.5 & Ordovician/Maquoketa & Siltstone & $<2$ & 1 & 1.2 & 2.5 & 10.2 & $<1$ & $<2$ & $<2$ & $<0.1$ & 2.3 & $<0.5$ & 1.755 \\
\hline 340.5 & Ordovician/Galena & Dolomite & $<2$ & 5 & 1.6 & 1 & 4.8 & $<1$ & $<2$ & $<2$ & 0.1 & 0.73 & $<0.5$ & 1.694 \\
\hline
\end{tabular}

bgs $=$ below ground surface (ground surface is approximately 740 feet Mean Sea Level) 
Table of Background Chemicals from Soil and Rock Samples using Total Digestion Inductively Coupled Plasma

\begin{tabular}{|c|c|c|c|c|c|c|c|c|c|c|c|c|}
\hline $\begin{array}{c}\text { Depth } \\
\text { (feet bgs) }\end{array}$ & Unit/Formation & Material Type & $\begin{array}{r}\mathrm{Sr} \\
\mathrm{ppm} \\
\end{array}$ & $\begin{array}{r}\text { Be } \\
\mathrm{ppm} \\
\end{array}$ & $\begin{array}{r}\mathrm{V} \\
\mathrm{ppm} \\
\end{array}$ & $\begin{array}{r}\mathrm{Cu} \\
\mathrm{ppm} \\
\end{array}$ & $\begin{array}{r}\mathrm{Pb} \\
\mathrm{ppm} \\
\end{array}$ & $\begin{array}{r}\mathrm{Zn} \\
\mathrm{ppm} \\
\end{array}$ & $\begin{array}{r}\mathrm{Ag} \\
\mathrm{ppm} \\
\end{array}$ & $\begin{array}{r}\mathrm{Ni} \\
\mathrm{ppm} \\
\end{array}$ & $\begin{array}{r}\mathrm{Cd} \\
\mathrm{ppm}\end{array}$ & $\begin{array}{r}\mathrm{Bi} \\
\mathrm{ppm} \\
\end{array}$ \\
\hline $7-11$ & Quaternary & Diamicton & 92 & 2 & 83 & 21 & 17 & 49 & $<0.4$ & 40 & $<0.5$ & $<5$ \\
\hline $11-14$ & Quaternary & Diamicton & 96 & 1 & 71 & 19 & 16 & 52 & $<0.4$ & 29 & $<0.5$ & $<5$ \\
\hline $28-35$ & Quaternary & Resedimented & 113 & 2 & 98 & 20 & 17 & 56 & $<0.4$ & 48 & $<0.5$ & $<5$ \\
\hline $39-42$ & Quaternary & Diamicton & 92 & 1 & 56 & 18 & 11 & 42 & $<0.4$ & 18 & $<0.5$ & $<5$ \\
\hline $42-53$ & Quaternary & Diamicton & 116 & 2 & 99 & 23 & 14 & 56. & $<0.4$ & 47 & 0.5 & 5 \\
\hline $54-58$ & Quaternary & Diamicton & 99 & $<1$ & 50 & 22 & 7 & 45 & 0.6 & 29 & 0.7 & $<5$ \\
\hline $58-65$ & Quaternary & Diamicton & 110 & $<1$ & 27 & 12 & 7 & 22 & $<0.4$ & 18 & $<0.5$ & $<5$ \\
\hline 69.0 & Silurian/Niagran & Dolomite & 83 & $<1$ & 8 & 3 & $<5$ & 12 & $<0.4$ & 3 & $<0.5$ & $<5$ \\
\hline 94.5 & Silurian/Niagran & Dolomite & 90 & $<1$ & 12 & 5 & 6 & 10 & $<0.4$ & 7 & $<0.5$ & $<5$ \\
\hline 105.0 & Silurian/Niagran & Dolomite & 89 & $<1$ & 12 & 6 & $<5$ & 162 & $<0.4$ & 10 & $<0.5$ & $<5$ \\
\hline 107.0 & Silurian/Niagran & Dolomite & 109 & $<1$ & 15 & 4 & $<5$ & 10 & $<0.4$ & 10 & $<0.5$ & $<5$ \\
\hline 130.0 & Silurian/Alexandrian & Dolomite & 95 & $<1$ & $<5$ & 3 & $<5$ & 9 & $<0.4$ & 2 & $<0.5$ & $<5$ \\
\hline 138.0 & Silurian/Alexandrian & Dolomite & 99 & $<1$ & 8 & 2 & $<5$ & 11 & $<0.4$ & 8 & $<0.5$ & $<5$ \\
\hline 150.0 & Silurian/Alexandrian & Dolomite & 121 & 2 & 109 & 21 & 8 & 13 & $<0.4$ & 29 & $<0.5$ & $<5$ \\
\hline 160.5 & Silurian/Alexandrian & Dolomite & 73 & $<1$ & 9 & 4 & $<5$ & 9 & $<0.4$ & 7 & $<0.5$ & $<5$ \\
\hline 162.0 & Silurian/Alexandrian & Dolomite & 70 & $<1$ & 16 & 6 & $<5$ & 15 & $<0.4$ & 11 & $<0.5$ & $<5$ \\
\hline 180.5 & Silurian/Alexandrian & Dolomite & 69 & $<1$ & 8 & 4 & $<5$ & 9 & $<0.4$ & 7 & $<0.5$ & $<5$ \\
\hline 194.5 & Ordovician/Maquoketa & Dolomite & 52 & $<1$ & $<5$ & 6 & $<5$ & 10 & $<0.4$ & 2 & $<0.5$ & $<5$ \\
\hline 208.5 & Ordovician/Maquoketa & Dolomite/Shale & 92 & $<1$ & 28 & 5 & $<5$ & 14 & $<0.4$ & 14 & $<0.5$ & $<5$ \\
\hline 268.5 & Ordovician/Maquoketa & Siltstone & 88 & $<1$ & 14 & 10 & $<5$ & 14 & $<0.4$ & 4 & $<0.5$ & $<5$ \\
\hline 340.5 & Ordovician/Galena & Dolomite & 57 & $<1$ & 7 & 8 & $<5$ & 17 & $<0.4$ & 2 & $<0.5$ & $<5$ \\
\hline
\end{tabular}

bgs $=$ below ground surface (ground surface is approximately 740 feet Mean Sea Level) 
Table of Background Major Elements from Soil and Rock Samples using Fusion ICP

\begin{tabular}{|c|c|c|c|c|c|c|c|c|c|c|c|c|c|c|}
\hline $\begin{array}{c}\text { Depth } \\
\text { (feet bgs) }\end{array}$ & Unit/Formation & Material Type & $\begin{array}{r}\mathrm{SiO}_{2} \\
\% \\
\end{array}$ & $\begin{array}{r}\mathrm{Al}_{2} \mathrm{O}_{3} \\
\% \\
\end{array}$ & $\begin{array}{r}\mathrm{Fe}_{2} \mathrm{O}_{3} \\
\% \\
\end{array}$ & $\begin{array}{c}\text { Mno } \\
\% \\
\end{array}$ & $\begin{array}{r}\mathrm{MbO} \\
\% \\
\end{array}$ & $\begin{array}{r}\mathrm{CaO} \\
\% \\
\end{array}$ & $\begin{array}{r}\mathrm{Na}_{2} \mathrm{O} \\
\% \\
\end{array}$ & $\begin{array}{r}\mathrm{K}_{2} \mathrm{O} \\
\% \\
\end{array}$ & $\begin{array}{c}\mathrm{TiO}_{2} \\
\% \\
\%\end{array}$ & $\begin{array}{r}\mathrm{P}_{2} \mathrm{O}_{5} \\
\% \\
\end{array}$ & $\begin{array}{r}\text { LOI }^{*} \\
\% \\
\end{array}$ & $\begin{array}{r}\text { TOTAL } \\
\% \\
\end{array}$ \\
\hline $7-11$ & Quaternary & Diamicton & 45.29 & 10.59 & 4.24 & 0.054 & 7.11 & 10.97 & 0.52 & 3.40 & 0.576 & 0.06 & 18.13 & 100.94 \\
\hline $11-14$ & Quaternary & Diamicton & 41.80 & 9.08 & 3.66 & 0.061 & 8.39 & 12.82 & 0.50 & 2.99 & 0.501 & 0.06 & 20.07 & 99.93 \\
\hline $28-35$ & Quaternary & Resedimented & 47.54 & 12.55 & 4.77 & 0.062 & 5.84 & 8.68 & 0.49 & 3.92 & 0.652 & 0.06 & 15.59 & 100.15 \\
\hline $39-42$ & Quaternary & Diamicton & 39.46 & 7.01 & 3.05 & 0.053 & 9.32 & 14.69 & 0.46 & 2.25 & 0.364 & 0.09 & 21.68 & 98.43 \\
\hline $42 \cdot 53$ & Quaternary & Diamicton & 44.01 & 12.33 & 4.33 & 0.055 & 6.81 & 9.52 & 0.42 & 3.58 & 0.575 & 0.09 & 16.93 & 98.65 \\
\hline $54-58$ & Quaternary & Diamicton & 40.66 & 5.14 & 3.58 & 0.083 & 10.46 & 16.30 & 0.65 & 1.37 & 0.274 & 0.09 & 20.16 & 98.77 \\
\hline $58-65$ & Quaternary & Diamicton & 35.50 & 4.51 & 1.77 & 0.041 & 11.53 & 17.59 & 0.53 & 1.55 & 0.276 & 0.09 & 25.42 & 98.81 \\
\hline 69.0 & Silurian/Niagran & Dolomite & 8.18 & 1.38 & 0.50 & 0.018 & 19.29 & 26.86 & 0.07 & 0.76 & 0.073 & 0.03 & 42.28 & 99.44 \\
\hline 94.5 & Silurian/Niagran & Dolomite & 15.69 & 3.06 & 0.98 & 0.043 & 16.61 & 23.47 & 0.06 & 1.54 & 0.159 & 0.04 & 37.19 & 98.84 \\
\hline 105.0 & Silurian/Niagran & Dolomite & 13.26 & 3.18 & 0.98 & 0.028 & 16.92 & 23.67 & 0.07 & 1.80 & 0.172 & 0.02 & 38.41 & 98.51 \\
\hline 107.0 & Silurian/Niagran & Dolomite & 11.63 & 2.85 & 0.95 & 0.028 & 17.55 & 24.69 & 0.07 & 1.51 & 0.144 & 0.02 & 39.19 & 98.63 \\
\hline 130.0 & Silurian/Alexandrian & Dolomite & 2.09 & 0.50 & 0.49 & 0.039 & 20.66 & 29.13 & 0.05 & 0.27 & 0.024 & 0.10 & 45.96 & 99.31 \\
\hline 138.0 & Silurian/Alexandrian & Dolomite & 5.46 & 1.33 & 0.63 & 0.035 & 20.11 & 28.55 & 0.05 & 0.59 & 0.082 & $<0.01$ & 41.64 & 98.48 \\
\hline 150.0 & Silurian/Alexandrian & Dolomite & 63.82 & 18.36 & 2.05 & 0.007 & 1.92 & 0.72 & 0.14 & 5.07 & 0.923 & 0.07 & 6.98 & 100.06 \\
\hline 160.5 & Silurian/Alexandrian & Dolomite & 3.04 & 0.26 & 0.58 & 0.018 & 20.94 & 29.30 & 0.06 & 0.07 & 0.012 & 0.01 & 44.80 & 99.09 \\
\hline 162.0 & Silurian/Alexandrian & Dolomite & 3.41 & 0.47 & 1.48 & 0.022 & 20.85 & 29.17 & 0.06 & 0.16 & 0.029 & 0.01 & 43.03 & 98.69 \\
\hline 180.5 & Silurian/Alexandrian & Dolomite & 5.84 & 1.27 & 0.73 & 0.043 & 19.53 & 27.62 & 0.05 & 0.51 & 0.066 & 0.05 & 43.09 & 98.80 \\
\hline 194.5 & Ordovician/Maquoketa & Dolomite & 1.70 & 0.35 & 1.21 & 0.109 & 19.96 & 29.41 & 0.06 & 0.10 & 0.016 & 0.28 & 45.25 & 98.45 \\
\hline 208.5 & Ordovician/Maquoketa & Dolomite/Shale & 23.96 & 4.75 & 1.42 & 0.031 & 14.03 & 20.33 & 0.10 & 1.86 & 0.242 & 0.32 & 31.81 & 98.85 \\
\hline 268.5 & Ordovician/Maquoketa & Siltstone & 18.31 & 2.14 & 1.75 & 0.049 & 16.36 & 23.73 & 0.07 & 0.78 & 0.104 & 0.14 & 34.35 & 97.78 \\
\hline 340.5 & Ordovician/Galena & Dolomite & 1.79 & 0.49 & 1.06 & 0.056 & 20.97 & 29.30 & 0.06 & 0.23 & 0.029 & 0.06 & 44.43 & 98.48 \\
\hline
\end{tabular}

* Lost on Ignition (LOI) is a measure of the $\mathrm{CO} 2$ and moisture content. This is usually high in MgO and $\mathrm{CaO}$ deposits.

bgs $=$ below ground surface (ground surface is approximately 740 feet Mean Sea Level) 